\title{
Veratric acid inhibits iNOS expression through the regulation of PI3K activation and histone acetylation in LPS-stimulated RAW264.7 cells
}

\author{
WOO-SUK CHOI ${ }^{1}$, YONG-BAE SEO ${ }^{1}$, PYUNG-GYUN SHIN ${ }^{2}$, WOE-YEON KIM ${ }^{3}$, \\ SANG YEOL LEE ${ }^{3}$, YOUNG-JU CHOI ${ }^{4}$ and GUN-DO KIM ${ }^{1}$

\begin{abstract}
${ }^{1}$ Department of Microbiology, College of Natural Sciences, Pukyong National University, Busan 608-737;
${ }^{2}$ Mushroom Research Division, National Institute of Horticultural and Herbal Science, Rural Development Administration, Suwon 441-707; ${ }^{3}$ Division of Applied Life Science, Plant Molecular Biology and Biotechnology Research Center, Gyeongsang National University, Jinju 660-701; ${ }^{4}$ Department of Food and Nutrition,
\end{abstract} \\ College of Medical Life, Silla University, Busan 617-736, Republic of Korea
}

Received April 21, 2014; Accepted October 9, 2014

DOI: $10.3892 /$ ijmm.2014.1982

\begin{abstract}
In the present study, we investigated regulatory effects of veratric acid on the production of nitric oxide (NO) in lipopolysaccharide (LPS)-stimulated RAW264.7 cells. NO production was significantly decreased by veratric acid in the LPS-stimulated RAW264.7 cells in a dose-dependent manner. The reduction in nitric oxide production was induced by the downregulation of inducible NO synthase (iNOS) expression. Veratric acid suppressed the LPS-induced effects on the regulatory and catalytic subunits of phosphoinositide 3-kinase (PI3K), comprised of p85, p110 $\alpha, \mathrm{p} 110 \beta$ and Akt. The acetylation of $\mathrm{p} 300$ and the phosphorylation of activating transcription factor 2 (ATF-2) induced by LPS were downregulated following treatment with veratric acid; similar effects were observed following treatment with LY294002, a specific inhibitor of PI3K/Akt. The LPS-induced expression of histone deacetylase (HDAC)3 decreased to basal levels following treastment with veratric acid, and its expression was also downregulated by LY294002. In the measurement of histone acetylation levels, the LPS-stimulated acetylation of histone $\mathrm{H} 4$ was significantly attenuated by veratric acid, and was also reduced following the inhibition of PI3K/Akt with LY294002. From our data, it can be concluded that veratric acid exerts a regulatory effect on LPS-induced iNOS expression. Our results suggest that veratric acid impedes the PI3K/Akt-mediated histone acetyltransferase (HAT) activation and HDAC expression induced by LPS, thereby abrogating iNOS expression.
\end{abstract}

Correspondence to: Professor Gun-Do Kim, Department of Microbiology, College of Natural Sciences, Pukyong National University, 45 Yongso-ro, Nam-gu, Busan 608-737, Republic of Korea E-mail: gundokim@pknu.ac.kr

Key words: veratric acid, inducible nitric oxide synthase, phosphoinositide 3-kinase/Akt, histone deacetylases, histone acetyltransferases, histone

\section{Introduction}

Lipopolysaccharide (LPS), a component of Gram-negative bacteria, induces the production of nitric oxide (NO) in monocytes and macrophages. NO plays a vital role in the regulation of inflammatory responses. In the acute inflammatory response, the increase in NO production represents the upregulation of the immune defense against pathogens. As NO induction enhances pro-inflammatory responses, in this manner, external invaders can be effectively excluded $(1,2)$. To produce NO during inflammation, the activation of inducible NO synthase (iNOS) is required. iNOS is a member of the NOS family, including endothelial NOS (eNOS) and neuronal NOS (nNOS). In inflammatory responses, LPS and various cytokines stimulate iNOS expression in macrophages. In particular, interleukin (IL)-4 with anti-CD23, interferon (IFN)- $\gamma$ with LPS and IFN- $\alpha / \beta$ activates iNOS, producing NO during the inflammatory response through the uptake of L-arginine $(3,4)$. Thus, the regulation of iNOS may prove to be a therapeutic strategy for the treament of inflammatory disorders (5).

During NO production, class $\mathrm{I}_{\mathrm{A}}$ phosphoinositide 3-kinase (PI3K) is associated with the regulation of iNOS expression. PI3Ks are divided into 4 classes according to their structure and substrate specificity. Class $\mathrm{I}_{\mathrm{A}} \mathrm{PI} 3 \mathrm{~K}$ is composed of the p85 regulatory subunit and the p110 catalytic subunit, and is a component of receptor-mediated signaling in the immune system (6). In receptor-mediating signaling, class $\mathrm{I}_{\mathrm{A}} \mathrm{PI} 3 \mathrm{~K}$ catalyzes the conversion of $\mathrm{PI}(4,5) \mathrm{P}_{2}$ into $\mathrm{PI}(3,4,5) \mathrm{P}_{3}$. In turn, $\mathrm{PI}(3,4,5) \mathrm{P}_{3}$ induces the phosphorylation of 3-phosphoinositide dependent protein kinase-1 (PDK-1) and the upregulation of Akt phosphorylation. The phosphorylation of Akt can be inhibited by the activation of phosphatase and tensin homologue (PTEN), converting $\mathrm{PIP}_{3}$ into $\mathrm{PIP}_{2}$ (7). It has been reported that $\mathrm{PI} 3 \mathrm{~K}$ is associated with the regulation of iNOS expression. PI3K/Akt signaling plays a role in the suppression of iNOS expression. The inhibition of PI $3 \mathrm{~K}$ in $\mathrm{C}_{6}$ glial cells enhances iNOS expression under LPS stimulation comparable with the LPS stimulation in wild-type $\mathrm{C}_{6}$ glial cells, and LPS-induced 
iNOS amplification following the inhibition of PI3K is not mediated by mitogen-activated protein kinase (MAPK) and nuclear factor $(\mathrm{NF})-\kappa \mathrm{B}(8,9)$. By contrast, the PI3K/Akt pathway upregulates iNOS expression. Treatment of hepatocytes with IL-1 $\beta$ has been shown to accentuate iNOS expression, but does not induce iNOS expression when PI3K/Akt is inhibited (10).

Histone acetyltransferases (HATs) and histone deacetylases (HDACs) are enzymes that mediate acetylation and deacetylation at lysine residues of various proteins, including histones (11). HATs are divided into 5 families: the GCN5related $N$-acetyltransferase (GNAT) family, represented by general control non-derepressible 5 (GCN5) and p300/CREBbinding protein (CBP)-associated factor (PCAF); the p300/CBP family, including p300 and CBP; the MYST family, including TAT-interacting protein 60 (Tip60); general transcription factor HATs; and nuclear hormone-related HATs (12). HDACs are classified into 4 classes containing a total of 18 enzymes. There is sequence similarity between classical HDACs (class I, II and IV) whose activity is dependent on $\mathrm{Zn}^{2+}$. Class III includes the family of sirtuins. In contrast to classical HDACs, the activity of class III sirtuins is dependent on $\mathrm{NAD}^{+}(13)$. The acetylation of histones by HATs and the tight package of chromatin structure by HDACs are critical to the control of gene expression. In resting cells, DNA is wound around an octomer with 2 molecules each of the core histone proteins, including $\mathrm{H} 2 \mathrm{~A}, \mathrm{H} 2 \mathrm{~B}, \mathrm{H} 3$ and H4. This chromatin structure suppresses gene expression as the basal transcription complex is unable to bind to DNA. Conversely, once the cells are activated by exogenous stimulation, lysine residues of a long terminal of each core histone are acetylated by HATs, including CBP, p300 and CBP/p300 co-activators, reducing the electrical charge of core histones. Thereby, the chromatin structure is transformed from the closed form to the opened conformation, allowing the binding of RNA polymerase II and the initiation of transcription (14). By contrast, removal of acetyl group by HDAC repacks chromatin and causes gene silencing (15). Thus, histone acetylation is closely linked to the induction of proinflammatory gene expression, and is considered a promising target for the treatment of inflammatory diseases.

Veratric acid is a phenolic compound derived from Sparassis crispa and has been reported to have antioxidant properties $(16,17)$. In our previous study, we proved that veratric acid inhibited iNOS expression through the inactivation of MAPKs and NF- $\kappa$ B in LPS-stimulated RAW264.7 cells (18). In the present study, we demonstrate that the inhibitory effects of veratric acid on the PI3K/Akt pathway, HATs and HDACs lead to the downregulation of iNOS expression in LPS-stimulated RAW264.7 cells.

\section{Materials and methods}

Chemicals and reagents. Veratric acid, LPS (Escherichia coli 0111:B4), Griess reagent and Tween-20 were purchased from Sigma-Aldrich (St. Louis, MO, USA). Skim milk powder was purchased from Bioshop Canada Inc. (Burlinton, ON, Canada). Dulbecco's modified Eagle's medium (DMEM), fetal bovine serum (FBS) and penicillin/streptomycin were purchased from Cellgro.com, Mediatech Inc. (Manassas, VA, USA). LY294002, a PI3K inhibitor, was purchased from Cayman Chemical Co. (Ann Arbor, MI, USA).
Animal experiments. All animal experiments were approved by the Institutional Animal Care and Use Committe of Dong-eui University, Busan, Korea. Female Balb/c mice (4-5 weeks of age, weighing 25-30 g) were purchased from Samtako Bio Korea Co. (Gyeonggi-do, Korea). These mice were housed in a specific pathogen-free facility with appropriate temperature and humidity and allowed free access to food and water. Skin inflammation was induced topically with the application of 12-O-tetradecanoylphorbol-13-acetate (TPA) on the dorsal skin of the mice; hair had been previously removed with an animal clipper. Briefly, the mice (5 per group) were either untreated, exposed to $200 \mu \mathrm{l}$ acetone, TPA alone or treated with 0.5 and $1 \mathrm{mg}(20,40$ and $80 \mathrm{mg} / \mathrm{kg}$ ) veratric acid (VA) in acetone $1 \mathrm{~h}$ prior to exposure to TPA. Following exposure and treatment as described above for $24 \mathrm{~h}$, the mice were sacrificed by cervical dislocation, and the dorsal skin was collected and snap-frozen in liquid nitrogen.

Cell culture. The RAW264.7 cell line was purchased from the American Tissue Culture Collection (ATCC, Manassas, VA, USA). The BV-2 microglial cell line was a generous gift from Professor Jong-Hwan Lee of Dong-eui University, Busan, Korea. The cells were maintained in Dulbecco's modified Eagle's medium (DMEM) supplemented with $10 \%$ fetal bovine serum (FBS), $100 \mathrm{U} / \mathrm{ml}$ penicillin and $100 \mu \mathrm{g} / \mathrm{ml}$ streptomycin, and were incubated under humidified conditions of $5 \% \mathrm{CO}_{2}$ at $37^{\circ} \mathrm{C}$. For the experiments, the cells were washed with warm DMEM and treated with with 100 or $200 \mu \mathrm{M}$ of veratric acid for $1 \mathrm{~h}$ prior to LPS stimulation. For the inhibition of PI3K, the cells were treated with the PI3K inhibitor, LY294002, for $1 \mathrm{~h}$ prior to the addition of veratric acid and LPS.

NO production. NO production in the RAW264.7 and BV-2 cell culture medium was measured using Griess reagent by reaction with nitrite. The cells $\left(5 \times 10^{5}\right.$ cells/well) were seeded in 24-well plates and incubated for $24 \mathrm{~h}$. Following incubation, the cells were pre-treated with veratric acid for $1 \mathrm{~h}$ prior to LPS stimulation for $24 \mathrm{~h}$. The cultured supernatant $(100 \mu \mathrm{l})$ was reacted with the same volume of Griess reagent $[1 \%$ sulfanilamide/ $0.1 \% \mathrm{~N}$-(1-naphthyl)-ethylenediamine dihydrochloride $/ 2.5 \% \mathrm{H}_{3} \mathrm{PO}_{4}$ ] for $10 \mathrm{~min}$ at room temperature. Nitrite was measured at $540 \mathrm{~nm}$ using an ELISA microplate reader (Molecular Devices, Sunnyvale, CA, USA).

RNA isolation and RT-PCR. The RAW264.7 cells were seeded on 12-well plates at a density of $2 \times 10^{5}$ cells/well and incubated $24 \mathrm{~h}$. The cells were cultured with or without $200 \mu \mathrm{M}$ of veratric acid for $1 \mathrm{~h}$ prior to LPS stimulation. Total RNA was isolated from the cells using the RNAeasy kit (Qiagen, Hilden, Germany, USA) . One microgram of total RNA was used for cDNA synthesis using AccuPower ${ }^{\circledR}$ RT PreMix (Bioneer Corp., Daejeon, Korea) containing M-MLV reverse transcriptase. The iNOS and GAPDH genes were amplified from the cDNA by PCR. The PCR primers used were as follows: iNOS (5'-ATGTCCGAAGCAAACATCAC-3' and 5'-TAATGTCC AGGAAGTAGGTG-3'); GAPDH (5'-AGGCCGGTGCT GAGTATGTC-3' and 5'-TGCCTGCTTCACCACCTTCT-3'); HDAC1 (5'-CGCATGACTCACAATTTGCT-3' and 5'-AAAC ACCGGACAGTCCTCAC-3'); HDAC2 (5'-AGACTGCAGTT GCCCTTGAT-3' and 5'-TTTGAACACCAGGTGCATGT-3'); 
and HDAC3 (5'-CACCTTTTCCAGCCAGTCAT-3' and 5'-GTA GCCACCACCTCCCAGTA-3'). The amplified DNA was electrophoresed on an agarose gel.

Nuclear extraction. The nuclear extraction was conducted using NE-PER nuclear and cytoplasmic extraction reagents according to the manufacturer's instructions (Pierce, Rockford, IL, USA). Briefly, the RAW264.7 cells were plated in 100-mm dishes, treated with veratric acid, stimulated with LPS for $1 \mathrm{~h}$, scraped into $3 \mathrm{ml}$ of cold PBS and pelleted by centrifugation. The cell pellets were resuspended in ice-cold CER I buffer and incubated on ice for $10 \mathrm{~min}$. The cells were then mixed with ice-cold CER II buffer, and centrifuged at $12,000 \mathrm{x} \mathrm{g}$ for $5 \mathrm{~min}$. The supernatant was transferred to a pre-chilled tube. The insoluble fraction was resuspended in ice-cold NER buffer, incubated on ice for $40 \mathrm{~min}$, and centrifuged at $12,000 \mathrm{x} \mathrm{g}$ for $10 \mathrm{~min}$. The supernatant fraction was used for analyzing the nuclear protein expression.

Western blot analysis. For preparing cell lysates, the RAW264.7 cells were treated with veratric acid for $1 \mathrm{~h}$ and then stimulated with LPS for the indicated periods of time. Following stimulation, the cells were washed twice with cold PBS and scraped off. The scraped cells were centrifuged and lysed with lysis buffer [50 mM Tris-Cl (pH 7.5), $150 \mathrm{mM} \mathrm{NaCl}, 1 \mathrm{mM}$ DTT, $0.5 \%$ NP-40, $1 \%$ Triton X-100, $1 \%$ deoxycholate, $0.1 \%$ SDS, $1 \mathrm{mM}$ PMSF, $1 \mathrm{mM}$ EDTA, $1 \mu \mathrm{M}$ aprotinin, $1 \mu \mathrm{M}$ leupeptin and $1 \mu \mathrm{M}$ pepstatin $\mathrm{A}]$ (Intron Biotechnology, Inc., Seongnam, Korea). For obtaining skin tissue lysates, at the end of the desired treatments, tissue lysates were prepared in denaturing buffer. Insoluble materials were discarded by centrifugation at $14,000 \mathrm{rpm}$ for $20 \mathrm{~min}$ at $4^{\circ} \mathrm{C}$. Protein content was measured using the Bradford method. Equal amounts of protein were separated on $12 \%$ SDS-polyacrylamide minigel. Proteins were transferred onto nitrocellulose membranes (Pall Corp., Ann Arbor, MI, USA) and subsequently blocked with 5\% skim milk and phosphate-buffered saline with Tween-20 (PBST; $135 \mu \mathrm{M}$ $\mathrm{NaCl}, 2.7 \mathrm{mM} \mathrm{KCl}, 4.3 \mathrm{mM} \mathrm{NAPO}_{4}, 1.4 \mathrm{mM} \mathrm{KH}_{2} \mathrm{PO}_{4}$ and $0.5 \%$ Tween-20) for $1 \mathrm{~h}$ at room temperature and probed overnight at $4^{\circ} \mathrm{C}$ with primary antibodies $(1: 1,000)$. Primary antibodies, including iNOS (\#2977), phosphorylted (p-)p85 (\#4228), p85 (\#4257), p110 $\alpha$ (\#4249), $110 \gamma$ (\#5405), p-PDK1 (\#3238), PDK1 (\#3062), p-Akt at Thr308 (\#2965), p-Akt at Ser473 (\#4058), Akt (\#9272), Ac-p300 (\#4771), p-activating transcription factor 2 (ATF-2; \#5112), PCAF (\#3378), HDAC1 (\#5356), HDAC2 (\#5513), HDAC3 (\#3949), Ac-H3 (\#9649), H3 (\#4499), Ac-H4 (\#2594), H4 (\#2935), lamin A (\#2032), GAPDH (\#2118) and $\beta$-actin (\#4967), were purchased from Cell Signaling Technology (Beverly, MA, USA). Primary antibodies to $110 \beta$ (sc-602), $110 \delta$ (sc-7176), p300 (sc-585), and ATF-2 (sc-187) were purchased from Santa Cruz Biotechnology Inc. (Santa Cruz, CA, USA). Primary antibodies were rabbit-derived antibodies apart from the primary antibodies to HDAC1, HDAC2 and HDAC 3 that were detected by anti-mouse IgG. After washing 3 times with PBST, the membranes were incubated with horseradish peroxidase-conjugated anti-rabbit IgG as secondary antibodies (1:10,000; Cell Signaling Technology) and washed 3 times with PBST. Immunoreactive bands were detected using an Enhanced chemiluminescence system detection system (Young In Frontier Co., Ltd., Seoul, Korea) and exposed to X-ray film.
Densiometric analysis was conducted using ImageJ software [National Institutes of Health (NIH), Bethesda, MD, USA].

Immunofluorescence staining. The expression of p-AKT in the RAW264.7 cells was detected by indirect immunofluorescence assay. The RAW264.7 macrophages were seeded on coverglass bottom dishes for $24 \mathrm{~h}$. The cells were cultured with or without $200 \mu \mathrm{M}$ of veratric acid for $1 \mathrm{~h}$ prior to stimulation with LPS for $3 \mathrm{~h}$. Following stimulation, the cells were stained with 4, 6diamidino-2-phenylindole (DAPI) (Roche Diagnostics Corp., Indianapolis, IN, USA) for $15 \mathrm{~min}$ and fixed with $4 \%$ paraformaldehyde (Junsei Chemical Co., Ltd., Tokyo, Japan). The fixed cells were blocked with 5\% mouse and rabbit serum (Santa Cruz Biotechnology Inc.) and polyclonal antibodies against p-AKT $(1 \mu \mathrm{g} / \mathrm{well})$ and $0.3 \%$ Triton X-100 were then applied for $1 \mathrm{~h}$. The cells were then incubated with anti-rabbit IgG tagged with Alexa Fluor 488 (Cell Signaling Technology) for $1 \mathrm{~h}$ and washed with PBS. The cells were embedded with ProLong Antifade Reagent (Invitrogen, Eugene, OR, USA). The samples were observed with an Nikon ECLIPSE 50i microscope equipped with a charged-coupled device (CCD) camera (Nikon, Tokyo, Japan). To determine subcellular regions of protein co-localization, individual red-, blue- and green-stained images derived from the same field were merged using High Content Analysis Software (Cambridge Healthtech Institute, Needham, MA, USA). The pixel intensities of the nucleus were measured as a percentage area of immunoreactivity with ImageJ software (NIH).

Statistical analyses. The data are expressed as the means \pm standard errors of the mean (SEM) of triplicate experiments. Each untreated control group and LPS-stimulated group was measured for statistically significant values by t-tests. To evaluate statistically significant values between the LPS-stimulated group and the veratric acid/LPS-treated group, ANOVA post hoc tests and Dunnett's multiple comparison tests were used. A P-value $<0.05$ was considered to indicate a statistically significant difference.

\section{Results}

Effects of veratric acid on iNOS expression in inflammatory cells and tissue. To evaluate the regulatory effects of veratric acid on iNOS expression, LPS-stimulated RAW264.7 cells and Balb/C mice with skin inflammation induced by TPA were treated with veratric acid. iNOS protein expression was then measured. In the RAW264.7 cells, the LPS-induced iNOS expression was suppressed by veratric acid in a dose-dependent manner (Fig. 1A). As shown by western blot analysis of the skin tissue lysates, the topical application of TPA onto mice transiently increased the iNOS protein level. However, treatment with veratric acid decreased the iNOS protein level in comparison with the TPA-treated mice (Fig 1B). Collectively, veratric acid exerts a regulatory effect on iNOS expression in inflammatory conditions.

Effects of veratric acid on the LPS-induced activation of the PI3K/Akt pathway. Previously, it has been demonstrated that the increase in iNOS expression in macrophages is mediate through the activation of PI3K and Akt (6). Thus, in this study, we analyzed regulatory effects of veratric acid on the LPS-induced activation of PI3K/Akt. Initially, the phosphory- 

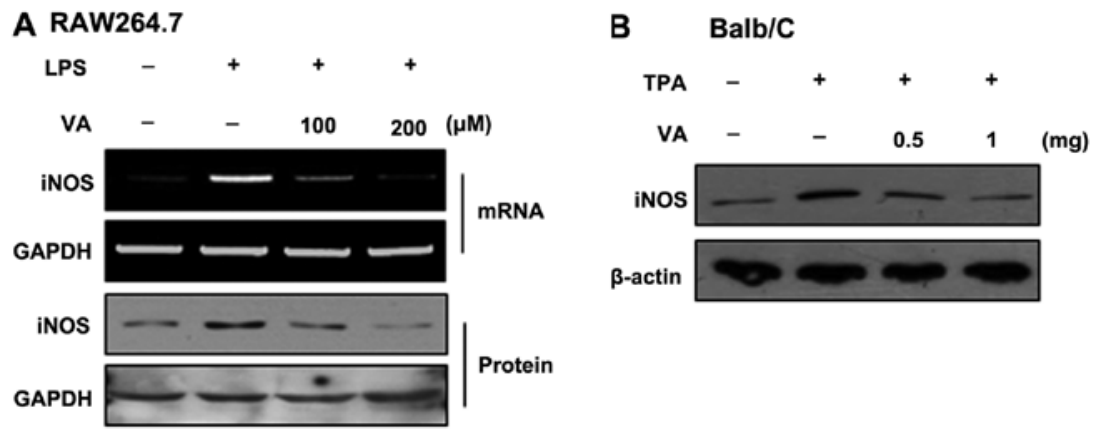

Figure 1. Effects of veratric acid on inducible nictric oxide synthase (iNOS) expression in lipopolysaccharide (LPS)-stimulated RAW264.7 cells and Balb/C mouse skins treated with 12- $O$-tetradecanoylphorbol-13-acetate (TPA). (A) iNOS levels in RAW264.7 cells treated with or without veratric acid (VA, 100 and $200 \mu \mathrm{M}$ ) for $1 \mathrm{~h}$ prior to LPS stimulation $(0.1 \mu \mathrm{g} / \mathrm{ml})$ for $12 \mathrm{~h}$. Each protein level was normalized by GAPDH. (B) iNOS levels in Balb/C mouse skins. Dorsal skin of mice was exposed topically to either $200 \mu \mathrm{l}$ of DMSO or TPA $(1 \mathrm{mg})$ in $200 \mu \mathrm{l}$ acetone, or treated with $0.5 \mathrm{and} 1 \mathrm{mg}$ veratric acid prior to $1 \mathrm{~h}$ of exposure to TPA. Each protein level was normalized to $\beta$-actin.
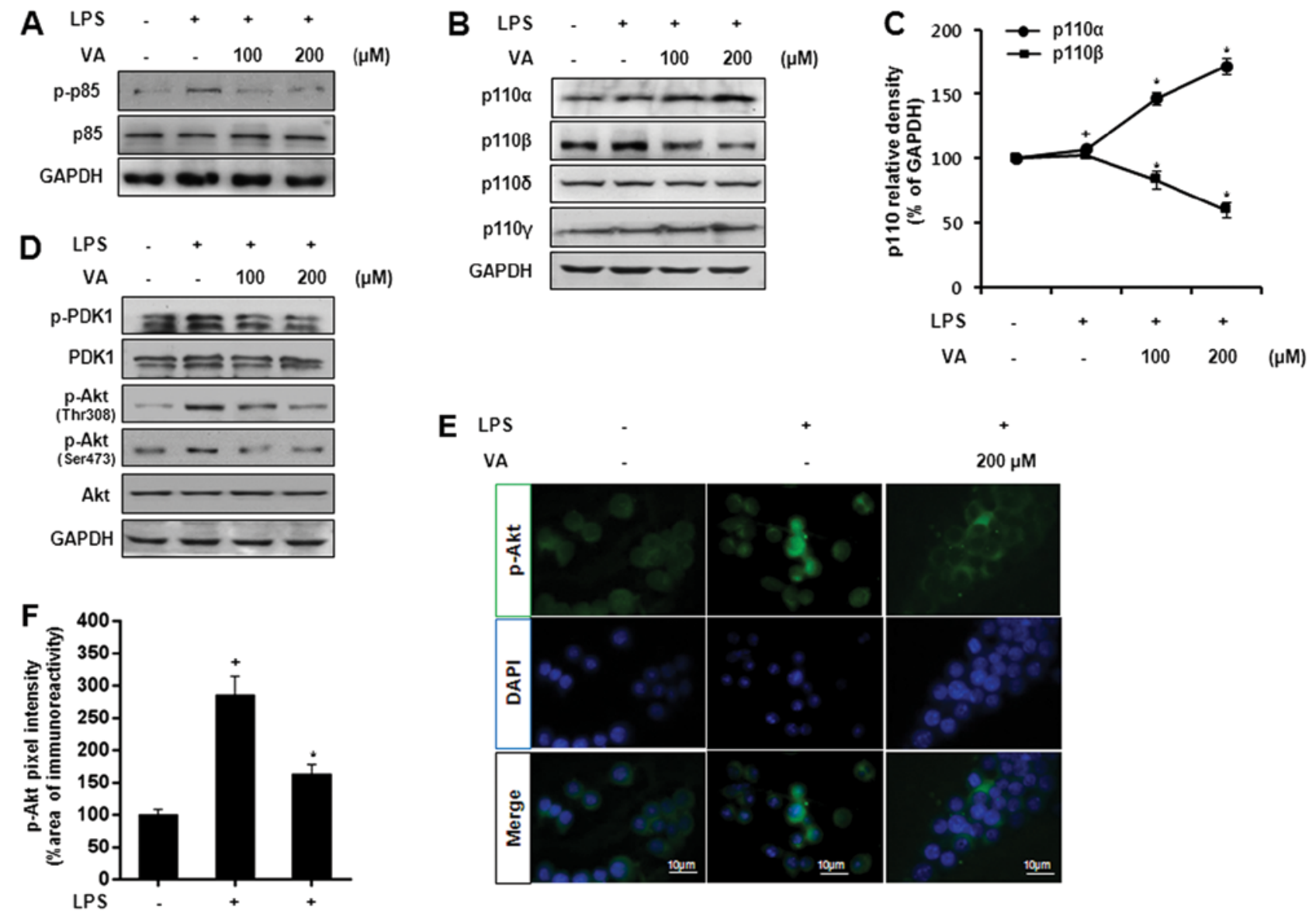

Figure 2. Effects of veratric acid on the PI3K/Akt pathway in LPS-stimulated RAW264.7 cells. (A) p85 phosphorylation levels in cells treated with or without veratric acid (VA) for $1 \mathrm{~h}$ prior to lipopolysaccharide (LPS) stimulation for $30 \mathrm{~min}$. (B) p110 subunit protein levels determined by western blot analysis. Cells were treated with or without veratric acid for $1 \mathrm{~h}$ prior LPS stimulation for $6 \mathrm{~h}$. (C) Densitometric analysis of p110 $\alpha$ and $\beta$; data are the means \pm SD of 3 experiments. (D) Phosphorylation levels of Akt and 3-phosphoinositide dependent protein kinase-1 (PDK-1) in cells treated with or without veratric acid for $1 \mathrm{~h}$ prior to LPS stimulation for $30 \mathrm{~min}$. (E) RAW264.7 cells were pre-treated with $200 \mu \mathrm{M}$ veratric acid for $1 \mathrm{~h}$ prior to stimulation with LPS for $3 \mathrm{~h}$. The expression of p-Akt was determined using an anti-p-Akt antibody and an anti-rabbit IgG tagged with Alexa Fluor 488, and cells were visualized using a Nikon ECLIPSE 50i microscope. (F) Pixel intensity of immunostained p-Akt was quantified using ImageJ software. Values were normalized to $100 \%$ for the expression of the untreated control group. Each value indicates the mean $\pm \mathrm{SD}$ of 3 independent experiments. Each protein level was normalized to GAPDH. ${ }^{+} \mathrm{P}<0.05$, control vs. LPS-treated group; ${ }^{P}<0.05$, veratric acid and LPS-treated group vs. LPS-treated group.

lation of p85, a regulatory subunit of PI3K, was measured at the protein level. As shown by western blot analysis, veratric acid decreased the LPS-induced $\mathrm{p} 85$ phosphorylation in a dose-dependent manner (Fig. 2A). In the same manner, veratric acid enhanced $\mathrm{p} 110 \alpha$ expression and decreased $\mathrm{p} 110 \beta$ expres- sion (Fig. 2B and C). As veratric acid regulated LPS-induced PI3K activation, we investigated the regulatory effects of veratric acid on PDK1 and Akt. As shown in Fig. 2D, veratric acid decreased the LPS-induced phosphorylation of PDK-1 and Akt. Immunofluorescenct staining revealed the transloca- 

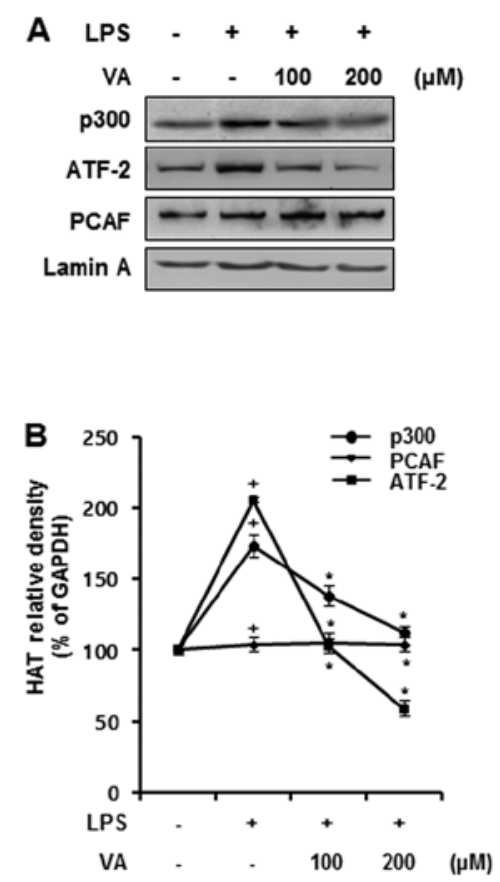
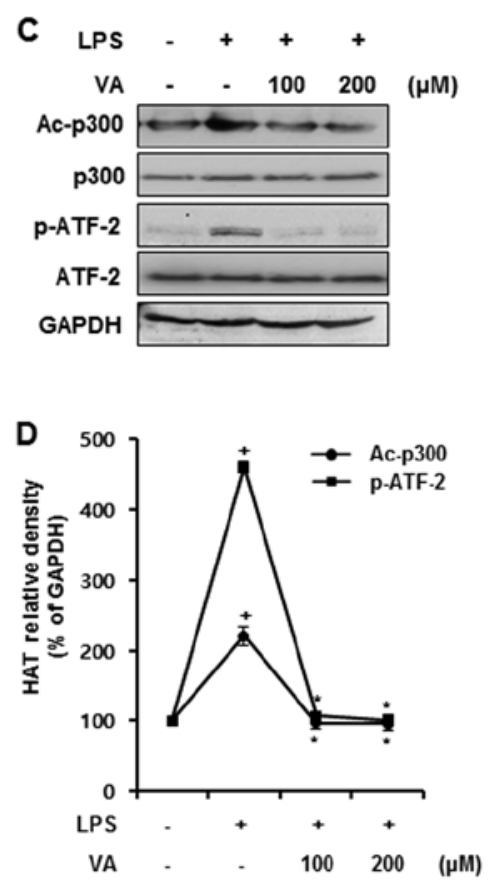

E
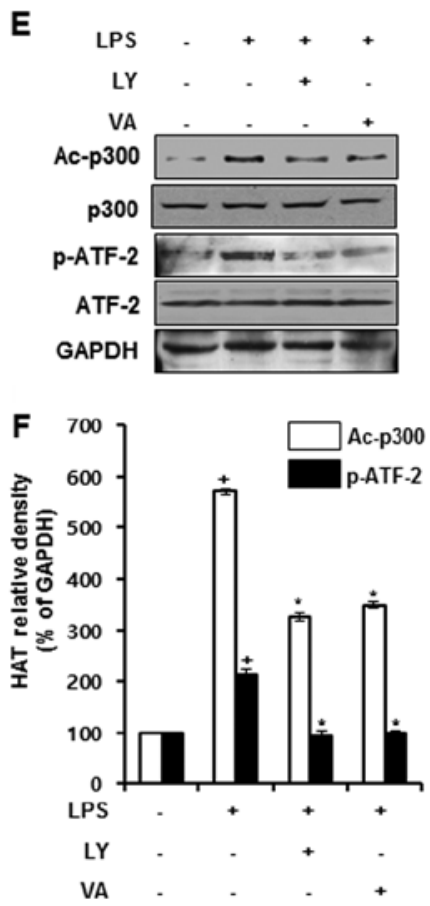

Figure 3. Effects of veratric acid on the expression and activation of histone acetyltransferases (HATs), including p300, p300/CREB-binding protein (CBP)associated factor (PCAF) and activating transcription factor 2 (ATF-2) in lipopolysaccharide (LPS)-stimulated RAW264.7 cells. (A) Nuclear extracts from cells treated with or without veraric acid (VA) for $1 \mathrm{~h}$ prior to LPS stimulation for $8 \mathrm{~h}$ were used to analyze expression of p300, PCAF and ATF-2. (Bottom panel) Densitometric analysis; data are the means \pm SD of 3 independent experiments. (B) Acetylated p300 (Ac-p300) and phosphorylated ATF-2 (p-ATF-2) in nuclear extracts were detected by western blot analysis. The nuclear extracts from cells treated with or without veratric acid for $1 \mathrm{~h}$ prior to LPS stimulation for $1 \mathrm{~h}$. (Bottom panel) Densitometric analysis; data are the means \pm SD of 3 independent experiments. (C) Ac-p300 and p-ATF-2 levels under treatment of veratric acid $(200 \mu \mathrm{M})$ or LY294002 (LY, $10 \mu \mathrm{M})$. Nuclear extracts from cells treated with or without veratric acid or LY294002 for $1 \mathrm{~h}$ prior to LPS stimulation for $1 \mathrm{~h}$ were used for western blot analysis. Columns represent the densitometric evaluation of 3 independent experiments (means \pm SD). Each protein level was normalized to lamin A. ${ }^{+} \mathrm{P}<0.05$, control vs. LPS-treated group; ${ }^{*} \mathrm{P}<0.05$, veratric acid and LPS-treated group vs. LPS-treated group.

tion of Akt from the cytosol to the nucleus in response to LPS, and this translocation was abolished by treatment with veratric acid (Fig. 2E and F). Taken together, our results indicate that veratric acid exerts inhibitory effects on the LPS-induced activation of the PI3K/Akt pathway in RAW264.7 cells.

Effects of veratric acid on the expression and activation of HATs in LPS-stimulated RAW264.7 cells. Akt activation promotes p300 HAT activity through its association with PCAF. The acetylation of p300 at lysine 1499 is crucial to enhancing its HAT activity, resulting in an increase in the acetylation of histone $\mathrm{H} 3$ or H4 (19,20). The phosphorylation of ATF-2 at threonine (Thr) 71 is regulated by PI3K/Akt signaling, and induces its HAT activity $(21,22)$. As we found that the LPS-induced activation of the PI3K/Akt pathway was regulated by veratric acid, we examined whether veratric acid regulates the activation and expression of HATs, including p300, ATF-2 and PCAF in the LPS-stimulated RAW264.7 cells. To examine the expression of the HATs, RAW264.7 cells were treated with veratric acid for $1 \mathrm{~h}$ prior to LPS stimulation for $12 \mathrm{~h}$. At the protein level, the expression of p300 and ATF-2 was increased by LPS stimulation, and reduced by veratric acid in a dosedependent manner. By contrast, PCAF expression was not significantly altered by LPS and veratric acid (Fig. 3A). As the expression of ATF-2 and p300 were sensitively regulated by veratric acid, we evaluated the regulatory effects of veratric acid on the acetylation of p300 and the phosphorylation of ATF-2. The cells were treated with veratric acid for $1 \mathrm{~h}$, and then stimulated with LPS for $1 \mathrm{~h}$. As shown in Fig. 3B, p300 acetylation and ATF-2 phosphorylation by LPS was observed, and this effect was inhibited by treatment with veratric acid in a concentration-dependent manner. As veratric acid inhibited the LPS-induced activation of the PI3K/Akt pathway and HAT activation, we wished to determine whether the inhibition of the $\mathrm{PI} 3 \mathrm{~K} / \mathrm{Akt}$ pathway is associated with the inactivation of $\mathrm{p} 300$ and ATF-2 in LPS-stimulated RAW264.7 cells. To reveal the correlation between the PI3K/Akt pathway and the activation of p300 and ATF-2, the PI3K/Akt pathway was inhibited by a specific inhibitor, LY294002, and the activity of HAT p300 and ATF-2 was examined by western blot analysis. In comparison with the LY294002-treated cells, the acetylation of p300 and the phosphorylation of ATF-2 were similarly downregulated in the veratric acid-treated cells (Fig. 3C). Thus, our results suggest that the LPS-induced activity of HAT p300 and ATF-2 is negatively regulated by veratric acid, induced by the suppression of the LPS-induced activation of the PI3K/Akt pathway.

Effects of veratric acid on HDAC expression in LPS-stimulated RAW264.7 cells. Chromatin remodeling events by HDAC are closely related to inflammatory gene transcription (23). To evaluate the effects of veratric acid on HDAC expression, we measured HDAC expression at the mRNA and protein level. The cells were treated with veratric acid for $1 \mathrm{~h}$ prior to LPS stimulation for $8 \mathrm{~h}$. As shown in Fig. $4 \mathrm{~A}$ and B, the mRNA expression of HDAC3 was increased by LPS stimulation, and was reduced to the basal level following treatment with veratric 

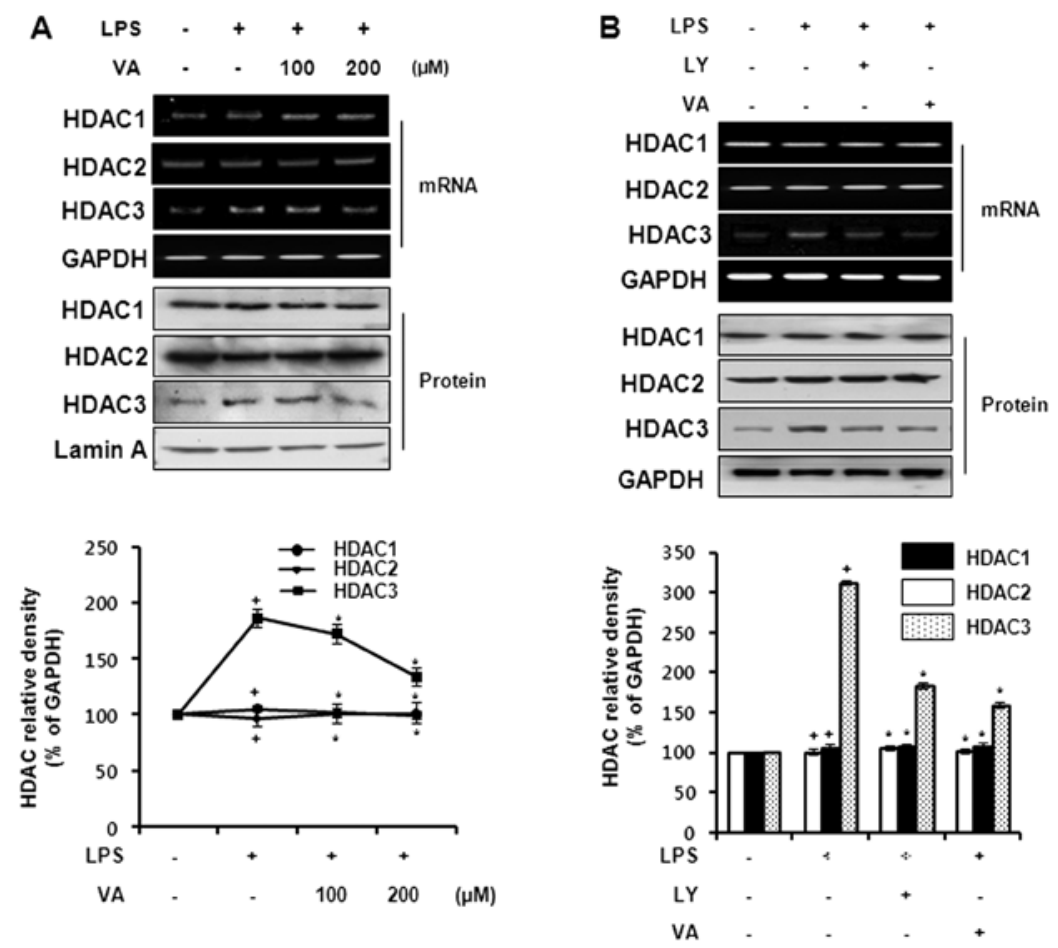

Figure 4. Effects of veratric acid on the expression of histone deacetylases (HDACs) in lipopolysaccharide (LPS)-stimulated RAW264.7 cells. (A) Nuclear extracts from cells treated with veratric acid (VA) for $1 \mathrm{~h}$ prior LPS stimulation for $8 \mathrm{~h}$ were used to detect the expression of HDAC1, HDAC2 and HDAC3 by western blot analysis. (Bottom panel) Densitometric analysis; data are the means \pm SD of 3 independent experiments. (B) Expression of HDAC1, HDAC2 and HDAC3 following treatment with veratric acid $(200 \mu \mathrm{M})$ or LY294002 (LY, $10 \mu \mathrm{M})$. Nuclear extracts from cells treated with or without veratric acid or LY294002 for $1 \mathrm{~h}$ prior to LPS stimulation for $8 \mathrm{~h}$ were used to determine HDAC1, HDAC2 and HDAC3 protein levels. Columns represent the densitometric evaluation of 3 independent experiments (means $\pm \mathrm{SD}$ ). Each protein level was normalized to lamin $\mathrm{A} .{ }^{+} \mathrm{P}<0.05$, control vs. LPS-treated group; ${ }^{*} \mathrm{P}<0.05$, veratric acid and LPS-treated group vs. LPS-treated group.

acid. Neither HDAC1 nor HDAC2 expression was influenced by LPS and veratric acid treatment. In accordance with the mRNA expression of HDACs, only HDAC3 was significantly regulated by veratric acid at the protein level. We also compared the expression of HDACs in veratric acid-treated cells with that in LY294002-treated cells. The cells were treated with veratric acid or LY294002 for $1 \mathrm{~h}$, and in turn stimulated with LPS for $8 \mathrm{~h}$. The LPS-induced expression of HDAC3 was downregulated by LY294002 and veratric acid; however, the expression of HDAC1 and HDAC2 was not affected in the same manner (Fig. 4A and B). As suggested by our results, the inhibitory effects of veratric acid on the LPS-stimulated HDAC3 expression are mediated through the PI3K/Akt signaling pathway.

Effects of veratric acid on LPS-induced histone acetylation. To confirm whether the regulation of HATs and HDACs by veratric acid is associated with histone acetylation, we measured the overall level of acetylation of histones at the protein level. The nuclear proteins were extracted from the cells treated with veratric acid for $1 \mathrm{~h}$ prior to LPS stimulation for $1 \mathrm{~h}$. LPS stimulated the acetylation of histone $\mathrm{H} 4$; this acetylation was downregulated by veratric acid in a dose-dependent manner. By contrast, no changes were observed in the acetylation levels of histone $\mathrm{H} 3$ following treatment with LPS and veratric acid (Fig. 5A). As treatment with LY294002 downregulated the acetylation of p300 and the phosphorylation of ATF-2, we hypothesized that LY294002 may attenuate the activity of HAT p300 and ATF-2. To demonstrate our hypothesis, we compared the acetylation levels of histone $\mathrm{H} 3$ and $\mathrm{H} 4$ in the LY294002treated cells and those treated with veratric acid by western blot analysis. LPS promoted the acetylation of histone $\mathrm{H} 4$, and this acetylation was downregulated by LY294002, which coincided with the acetylation levels of histone $\mathrm{H} 4$ in the veratric acidtreated cells (Fig. 5B). Hence, our results indicated that veratric acid suppressed the LPS-induced histone $\mathrm{H} 3$ acetylation, and suggested that the suppression of the LPS-induced histone H4 acetylation by veratric acid may be accompanied by the regulation of the PI3K/Akt pathway.

\section{Discussion}

In a previous study, we proved that veratric acid downregulates the LPS-induced expression of iNOS in RAW264.7 cells by inhibiting the activation of MAPKs and transcription factors, including signal transducer and activator of transcription (STAT)1, STAT3 and NF-кB (18). In the present study, we demonstrated that treatment with veratric acid suppressed the LPS-induced effects on p85, p110 $\beta$ and Akt in a dose-dependent manner. The acetylation levels of histone $\mathrm{H} 4$ were increased by LPS stimulation; however, following treatment with veratric acid, the acetylation levels returned to basal levels. The results from western blot analysis for HATs and HDACs provided additional evidence of the inhibitory effects of veratric acid on histone $\mathrm{H} 4$.

Class $\mathrm{I}_{\mathrm{A}}$ PI3K forms a heterodimer, composed of catalytic and regulatory subunits. There are 3 catalytic subunits, 

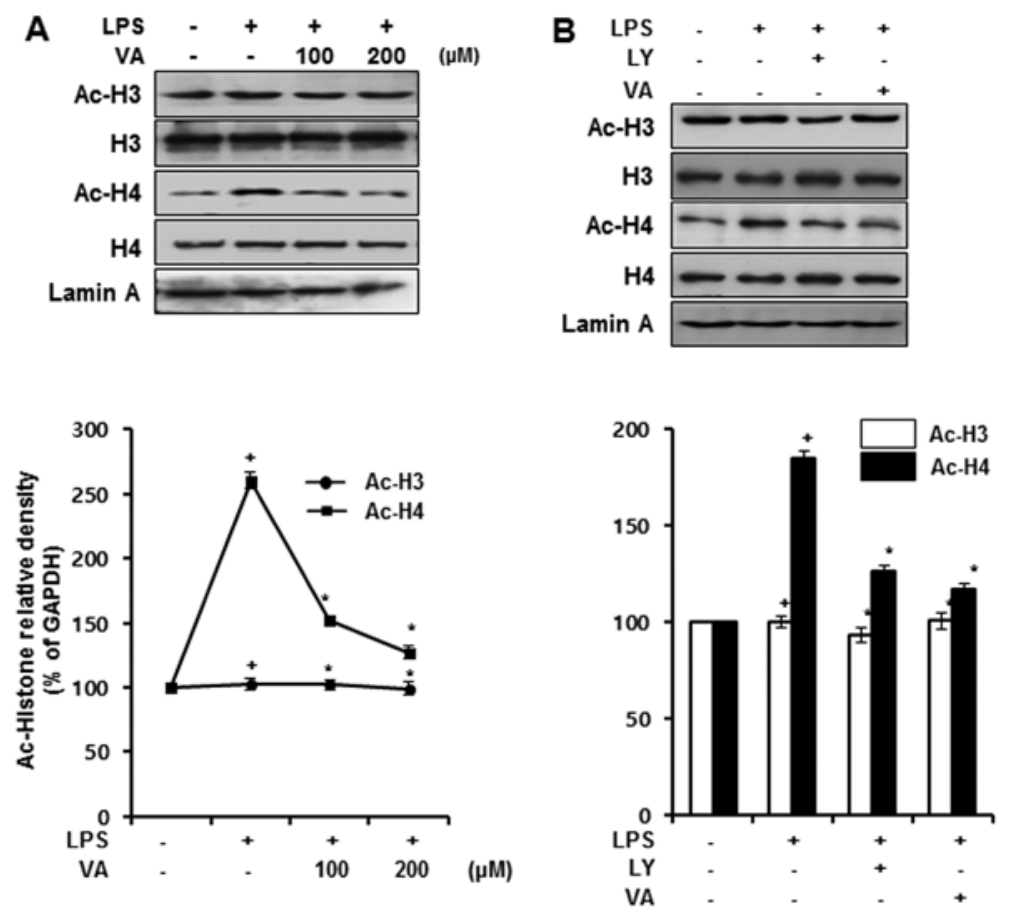

Figure 5. Effects of veratric acid on the acetylation of histone H3 and H4 in lipopolysaccharide (LPS)-stimulated RAW264.7 cells. (A) Protein levels of acetylated (Ac)-histone $\mathrm{H} 3$ and $\mathrm{H} 4$ in cells treated with or without veratric acid for $1 \mathrm{~h}$ prior to LPS stimulation for $1 \mathrm{~h}$. Nuclear extracts were used to determine the protein levels of Ac-histone H3 and H4. (Bottom panel) Densitometric analysis; data are the means \pm SD of 3 independent experiments. (B) Protein levels of Ac-histone H3 and H4 in cells treated with veratric acid $(200 \mu \mathrm{M})$ or LY294002 (LY, $10 \mu \mathrm{M})$. Nuclear extracts from cells treated with or without veratric acid or LY294002 for $1 \mathrm{~h}$ prior to LPS stimulation for $1 \mathrm{~h}$ were used to assess the levels of Ac-histone $\mathrm{H} 3$ and $\mathrm{H} 4$. Columns represent the densitometric evaluation of 3 independent experiments (means \pm SEM). Each protein level was normalized by lamin $\mathrm{A} .{ }^{+} \mathrm{P}<0.05$, control vs. LPS-treated group; ${ }^{\mathrm{P}} \mathrm{P}<0.05$, veratric acid and LPS-treated group vs. LPS-treated group.

including $\mathrm{p} 110 \alpha, \beta$ and $\delta$, and 5 regulatory subunits, including $\mathrm{p} 85 \alpha, \mathrm{p} 85 \beta, \mathrm{p} 55 \alpha, \mathrm{p} 55 \gamma$ and $\mathrm{p} 50 \alpha$. Class $\mathrm{I}_{\mathrm{B}} \mathrm{PI} 3 \mathrm{~K}$ is expressed preferentially in leukocytes, and is also composed of catalytic and regulatory subunits, p110 $\gamma$ and p101, respectively $(24,25)$. $\mathrm{PI} 3 \mathrm{~K}$ is recruited to the inner surface of the plasma membrane, and converts $\mathrm{PI}(4,5) \mathrm{P}_{2}$ into $\mathrm{PI}(3,4,5) \mathrm{P}_{3}$, which results in the recruitment and activation of PDK-1 at the plasma membrane. Akt is then phosphorylated by PDK-1 at Thr308 and activated (26). Our findings indicated that LPS stimulated the phosphorylation of p85, PDK-1 and Akt, and increased p110 $\beta$ expression. However, treatment with veratric acid reversed the LPS-induced effects on these proteins (Fig. 2A-C). It has been reported that $\mathrm{p} 85, \mathrm{p} 110$ and Akt are tightly linked to the regulation of iNOS expression. The catalytic subunits of PI3K, including $\mathrm{p} 110 \alpha$ and $\mathrm{p} 110 \beta$, regulate Akt phosphorylation in LPS-stimulated macrophages. In p110 $\alpha$-deficient macrophages, Akt is strikingly phosphorylated by LPS stimulation, whereas the LPS-induced Akt phosphorylation is not properly induced in p110 $\beta$-deficient macrophages (27). Sheu et al (28) demonstrated that PI3K is associated with the regulation of iNOS expression in mesangial cells. The LPS and IFN- $\gamma$-induced iNOS expression in mesangial cells was downregulated by the PI3K/Akt inhibitor, LY294002. Another recent study demonstrated that psoralidin suppresses the LPS-induced expression of p85 and Akt in RAW264.7 cells, resulting in the decrease of iNOS expression (29). Our findings indicated that the treatment of the skin of mice with inflammation induced by TPA and LPS-stimulated RAW264.7 cells with veratric acid attenuated iNOS expression (Fig. 1). Taken together, these observations suggested that veratric acid inhibited the LPS-induced Akt activation through the regulation of the LPS-induced effects on $\mathrm{p} 85, \mathrm{p} 110 \alpha$ and $\mathrm{p} 110 \beta$, thereby suppressing iNOS expression.

It has been reported that PI3K/Akt signaling is required to maintain the stability and HAT function of p300, closely related to iNOS expression. In LY294002-treated HeLa cells, p300 is not sufficiently expressed, and its transcriptional function related to retinoic acid receptor signaling is impaired, while stable cells expressing constitutive active Akt maintain the steady-state level and function of p300 in a similar manner (30). Likewise, Akt activation by suberoylanilide hydroxamic acid (SAHA)-induced HDAC inhibition stimulates p300 phosphorylation, resulting in the augmentation of NF- $\mathrm{KB}$ acetylation. Activated Akt, p300 and NF- $\mathrm{KB}$ are recruited to the NF-kB-regulated promoters, including cIAP-2 and Bfl-1/ A1 promoters. However, LY294002 treatment downregulates the SAHA-induced p300 phosphorylation, and in turn suppresses NF- $\mathrm{KB}$ acetylation (31). In macrophages, acetylated p300 elicits the acetylation of p50 in the nucleus, accentuating iNOS expression by increasing NF- $\mathrm{kB}$ binding to its corporate sites. Roscovitine, an inhibitor of cyclin E-CdK2, abrogates p300 HAT activity, and then impedes iNOS promoter activity induced by LPS/IFN- $\gamma$ (32). These findings underscore the distinct roles of Akt-mediated p300 acetylation in the modulation of iNOS expression. In this study, in accordance with the acetylation levels of p300 in the LY294002-treated cells, p300 acetylation in the veratric acid-treated cells returned to levels comparable to those observed in the control cells, suggesting that veartric acid blocks the LPS-induced p300 acetylation through the suppression of PI3K/Akt signaling (Fig. 3). Not only p300 acetylation, but also ATF-2 phosphorylation is 
associated with iNOS expression through its HAT function. It has been reported that ATF-2 phosphorylation at Thr71 induces HAT function. Under conditions of leucine deprivation, ATF-2 is rapidly phosphorylated; histone $\mathrm{H} 4$ and $\mathrm{H} 2 \mathrm{~B}$ are markedly acetylated, promoting the transcriptional activation of $\mathrm{CHOP}$, a CCAAT/enhancer-binding protein-related gene (33). ATF-2 phosphorylation at Thr71 enhances iNOS expression by advanced glycation end products (AGEs), while SB203580, a specific inhibitor of p38, suppresses the AGE-induced ATF-2 activation, resulting in a decrease in iNOS expression (34).PI3K/ Akt signaling also affects ATF-2 phosphorylation. In regenerating gene (Reg)-overexpressed $\beta$-cells, ATF- 2 phosphorylation is markedly amplified, increasing cyclin D1 promoter activity. However, LY294002 treatment returned the amplified ATF-2 phosphorylation to the basal level observed in the control group (21). Our findings demonstrated that the LPS-induced ATF-2 phosphorylation was attenuated by veratric acid and LY294002 (Fig. 3). Thus, the regulatory effects of veratric acid on $\mathrm{CBP} / \mathrm{p} 300$ acetylation and ATF-2 phosphorylation have important implications for the PI3K/Akt-mediated regulation of iNOS expression in LPS-stimulated macrophages.

We found that the LPS-induced expression of HDAC1 and HDAC3 was reduced following treatment with veratric acid, and there was no apparent change in HDAC2 expression folloiwng treatment with LPS and veratric acid (Fig. 4A). It has been reported that class I HDACs, including HDAC1, HDAC2 and HDAC3, closely correlate with iNOS expression. The specific knockdown of HDAC1, HDAC2 and HDAC3 in INS cells significantly reduces cytokine-induced iNOS expression, while the knockdown of only HDAC3 modulates the binding of $\mathrm{NF}-\kappa \mathrm{B}$ to the iNOS promoter (35). HDAC3 physically interacts with a NF- $\kappa \mathrm{B}$ p65 subunit in the nucleus, and deacetylates tumor necrosis factor (TNF)- $\alpha$-induced p65 acetylation, promoting the export of p65 from the nucleus to the cytoplasm (36). HDAC1 and HDAC2 also directly interact with the p65 protein of $\mathrm{NF}-\kappa \mathrm{B}$, exerting its corepressor function through the interaction and enhancing cytokine induction of iNOS promoter $(37,38)$. HDAC3 is closely related to the regulation of Akt phosphorylation. HDAC3 depletion inhibits TGF- $\beta$-induced Akt phosphorylation in murine fibroblasts (39), and reduces Akt phosphorylation in Drosophila which antagonizes PI3K-induced tissue overgrowth (40). These data suggest that HDAC1 and HDAC3 activity is required for iNOS expression through the regulation of the activation of Akt and NF- $\mathrm{NB}$. Our results, therefore, suggest that the inhibitory effects of veratric acid on the LPS-induced HDAC1 and HDAC3 expression may elicit the decrease in iNOS expression associated with the negative regulation of Akt phosphorylation.

Upon Toll-like receptor 4 (TLR4) activation, the HATs, GCN5 and PCAF, are required for the inducible acetylation of $\mathrm{H} 4 \mathrm{~K} 5 / \mathrm{K} 8 / \mathrm{K} 12$, resulting in the expression of primary response genes (PRGs) via the prompt activation of transcription factors, such as $\mathrm{NF}-\kappa \mathrm{B}$. In contrast to the inducible acetylation of histone $\mathrm{H} 4$ lysine residues by PCAF and GCN5, CBP/p300 are recruited to many $\mathrm{PRG}$ promoters in resting cells, and constitutively maintain low levels of $\mathrm{H} 3 \mathrm{~K} 9$ acetylation at the promoters (41). The activation of HAT p300 amplifies the acetylation of histone $\mathrm{H} 3$ and $\mathrm{H} 4$ at the ICAM-1 promoter in response to TNF- $\alpha$, facilitating PCAF recruitment to p300 and histone $\mathrm{H} 3$ acetylation (20). In the case of ATF-2, it selectively acetylates histone $\mathrm{H} 2 \mathrm{~B}$ and $\mathrm{H} 4$ through phosphorylation at Thr71 and K5, K8 and K16 residues in histone H4 are specifically acetylated by ATF-2 HAT activity (22). Studies have demonstrated the differential histone acetylation at lysine residues under inflammatory conditions. In a mouse model of mastitis and in a rat model of colitis induced by Escherichia coli, significant hyperacetylation at the histone $\mathrm{H} 4 \mathrm{~K} 8$ residue in inflammatory cells was observed. The histone H4K8 hyperacetylation is caused by the autoacetylation of p300, facilitating the expression of iNOS, MAPKs and inflammatory cytokines. Histone $\mathrm{H} 3 \mathrm{~K} 9$ acetylation levels were not significantly altered in the mastitis model, but the total acetylation levels of histone $\mathrm{H} 3$ in the colitis model were augmented $(42,43)$. The reason why the acetylation levels of histone $\mathrm{H} 3$ are differentially regulated in inflammatory diseases is that histone $\mathrm{H} 3$ acetylation does not markedly and rapidly responded to inflammatory signals comparable to histone $\mathrm{H} 4$ acetylation (44). Histone $\mathrm{H} 4$ is promptly acetylated by inflammatory stimuli, and the hyperacetylation of histone $\mathrm{H} 4$ at the iNOS promoter is associated with cytokine-induced iNOS expression. For the transcription of the iNOS gene, NF- $\mathrm{BB}$ should be bound to its promoter region, placed in -971 to -962 and -85 to -76 from the transcriptional start site of the murine $i N O S$ gene. The region -978 to -710 of the murine iNOS promoter contains a $\kappa \mathrm{B}$ enhancer element, which is wound by histone H4. In murine mesangial cells, the inhibition of PI3K and HDACs augments histone $\mathrm{H} 4$ acetylation at the iNOS promoter, inducing the reduction of cytokine-induced iNOS expression $(45,46)$. In this study, we detected a decrease in the LPS-induced H4K8 acetylation by veratric acid, and there was no significant alteration in $\mathrm{H} 3 \mathrm{~K} 9$ acetylation by LPS and veratric acid (Fig. 5), suggesting that the suppression of H4K8 acetylation may be involved in the inhibitory effects of veratric acid on iNOS expression. Although we evaluated the alteration in LPS-induced overall H4K8 acetylation by veratric acid, further studies are required to examine the veratric acid-mediated changes in the acetylation levels of histone $\mathrm{H} 4$ at the iNOS promoter.

In conclusion, veratric acid plays an inhibitory role in iNOS expression by inflammatory stimuli. Our results suggest that it suppresses the LPS-induced effects on $\mathrm{p} 85$, p110 $\alpha$ and $\mathrm{p} 110 \beta$, leading to Akt inactivation. The LPS-induced activity of HAT p300 and ATF-2 and the expression of HDAC3 are downregulated by veratric acid, thereby decreasing histone H4K8 acetylation. In addition, the regulation of protein activation and expression by veratric acid is consistent with that observed with treatment with LY294002, a PI3K inhibitor. The inhibitory effects of veratric acid on the PI3K/Akt pathway may thus elicit the suppression of histone $\mathrm{H} 4$ acetylation through the inactivation of HATs and HDACs, resulting in the downregulation of iNOS expression. Our data suggest that veratric acid has potential for use as a therapeutic agent for the transcriptional regulation of iNOS expression and may contribute to alleviating inflammatory diseases through the prevention of excessive NO production.

\section{Acknowledgements}

The present study was supported by the Next-Generation BioGreen 21 Program (SSAC, NO. PJ009615) from the Rural Development Administration. 


\section{References}

1. Swantek JL, Christerson L and Cobb MH: Lipopolysaccharideinduced tumor necrosis factor- $\alpha$ promoter activity is inhibitor of nuclear factor- $\kappa \mathrm{B}$ kinase-dependent. J Biol Chem 274: $11667-11671,1999$.

2. Sharma J, Al-Omran A and Parvathy S: Role of nitric oxide in inflammatory diseases. Inflammopharmacology 15: 252-259, 2007

3. Bogdan C: Nitric oxide and the immune response. Nat Immunol 2: 907-916, 2001

4. Bogdan C, Röllinghoff $M$ and Diefenbach A: The role of nitric oxide in innate immunity. Immunol Rev 173: 17-26, 2000.

5. Zamora R, Vodovotz Y and Billiar TR: Inducible nitric oxide synthase and inflammatory diseases. Mol Med 6: 347-373, 2000.

6. Sakai K, Suzuki H, Oda H, et al: Phosphoinositide 3-kinase in nitric oxide synthesis in macrophage: critical dimerization of inducible nitric-oxide synthase. J Biol Chem 281: 17736-17742, 2006.

7. Luyendyk JP, Schabbauer GA, Tencati M, Holscher T, Pawlinski R and Mackman N: Genetic analysis of the role of the PI3K-Akt pathway in lipopolysaccharide-induced cytokine and tissue factor gene expression in monocytes/macrophages. J Immunol 180: 4218-4226, 2008.

8. Pahan K, Raymond JR and Singh I: Inhibition of phosphatidylinositol 3-kinase induces nitric-oxide synthase in lipopolysaccharide-or cytokine-stimulated C6 glial cells. J Biol Chem 274: 7528-7536, 1999.

9. Pahan K, Liu X, Wood C and Raymond JR: Expression of a constitutively active form of phosphatidylinositol 3-kinase inhibits the induction of nitric oxide synthase in human astrocytes. FEBS Lett 472: 203-207, 2000.

10. Teshima S, Nakanishi H, Nishizawa M, et al: Up-regulation of IL-1 receptor through PI3K/Akt is essential for the induction of iNOS gene expression in hepatocytes. J Hepatol 40: 616-623, 2004.

11. Choudhary C, Kumar C, Gnad F, et al: Lysine acetylation targets protein complexes and co-regulates major cellular functions. Science 325: 834-840, 2009.

12. Ghizzoni M, Haisma HJ, Maarsingh $\mathrm{H}$ and Dekker FJ: Histone acetyltransferases are crucial regulators in NF- $\kappa \mathrm{B}$ mediated inflammation. Drug Discov Today 16: 504-511, 2011.

13. Yang WM, Tsai SC, Wen YD, Fejér G and Seto E: Functional domains of histone deacetylase-3. J Biol Chem 277: 9447-9454 2002.

14. Roth SY, Denu JM and Allis CD: Histone acetyltransferases. Annu Rev Biochem 70: 81-120, 2001.

15. Lin HY, Chen CS, Lin SP, Weng JR and Chen CS: Targeting histone deacetylase in cancer therapy. Med Res Rev 26: 397-413, 2006.

16. Raja B: Veratric acid, a phenolic acid attenuates blood pressure and oxidative stress in l-NAME induced hypertensive rats. Eur J Pharmacol 671: 87-94, 2011.

17. Kim MY, Seguin P, Ahn JK, et al: Phenolic compound concentration and antioxidant activities of edible and medicinal mushrooms from Korea. J Agr Food Chem 56: 7265-7270, 2008.

18. Choi WS, Shin PG, Lee JH and Kim GD: The regulatory effect of veratric acid on NO production in LPS-stimulated RAW264. 7 macrophage cells. Cell Immunol 280: 164-170, 2012.

19. Stiehl DP, Fath DM, Liang D, Jiang Y and Sang N: Histone deacetylase inhibitors synergize p300 autoacetylation that regulates its transactivation activity and complex formation. Cancer Res 67: 2256-2264, 2007.

20. Huang WC and Chen CC: Akt phosphorylation of p300 at Ser-1834 is essential for its histone acetyltransferase and transcriptional activity. Mol Cell Biol 25: 6592-6602, 2005.

21. Takasawa S, Ikeda T, Akiyama T, et al: Cyclin D1 activation through ATF-2 in Reg-induced pancreatic $\beta$-cell regeneration. FEBS Lett 580: 585-591, 2006.

22. Kawasaki H, Schiltz L, Chiu R, et al: ATF-2 has intrinsic histone acetyltransferase activity which is modulated by phosphorylation. Nature 405: 195-200, 2000

23. Miao F, Gonzalo IG, Lanting L and Natarajan R: In vivo chromatin remodeling events leading to inflammatory gene transcription under diabetic conditions. J Biol Chem 279: 18091-18097, 2004.

24. Geering B, Cutillas PR, Nock G, Gharbi SI and Vanhaesebroeck B Class IA phosphoinositide 3-kinases are obligate p85-p110 heterodimers. Proc Natl Acad Sci USA 104: 7809-7814, 2007.

25. Koyasu S: The role of PI3K in immune cells. Nat Immunol 4 313-319, 2003.
26. Monick MM, Carter AB, Robeff PK, Flaherty DM, Peterson MW and Hunninghake GW: Lipopolysaccharide activates Akt in human alveolar macrophages resulting in nuclear accumulation and transcriptional activity of $\beta$-catenin. J Immunol 166: 4713-4720, 2001

27. Tsukamoto K, Hazeki K, Hoshi M, et al: Critical roles of the p110 $\beta$ subtype of phosphoinositide 3-kinase in lipopolysaccharide-induced Akt activation and negative regulation of nitrite production in RAW 264.7 cells. J Immunol 180: 2054-2061, 2008.

28. Sheu ML, Chao KF, Sung YJ, Lin WW, Lin-Shiau SY and Liu SH: Activation of phosphoinositide 3-kinase in response to inflammation and nitric oxide leads to the up-regulation of cyclooxygenase-2 expression and subsequent cell proliferation in mesangial cells. Cell Signal 17: 975-984, 2005.

29. Chiou WF, Don MJ, Liao JF and Wei BL: Psoralidin inhibits LPS-induced iNOS expression via repressing Syk-mediated activation of PI3K-IKK-IкB signaling pathways. Eur J Pharmacol 650: 102-109, 2011

30. Chen J, Halappanavar S, St-Germain J, Tsang B and Li Q: Role of Akt/protein kinase B in the activity of transcriptional coactivator p300. Cell Mol Life Sci 61: 1675-1683, 2004.

31. Liu Y, Denlinger CE, Rundall BK, Smith PW and Jones DR: Suberoylanilide hydroxamic acid induces Akt-mediated phosphorylation of $\mathrm{p} 300$, which promotes acetylation and transcriptional activation of RelA/p65. J Biol Chem 281: 31359-31368, 2006.

32. Deng WG and Wu KK: Regulation of inducible nitric oxide synthase expression by p300 and p50 acetylation. J Immunol 171: 6581-6588, 2003.

33. Bruhat A, Chérasse Y, Maurin AC, et al: ATF2 is required for amino acid-regulated transcription by orchestrating specific histone acetylation. Nucleic Acid Res 35: 1312-1321, 2007.

34. Chang PC, Chen TH, Chang CJ, Hou CC, Chan P and Lee HM: Advanced glycosylation end products induce inducible nitric oxide synthase (iNOS) expression via a p38 MAPK-dependent pathway. Kidney Int 65: 1664-1675, 2004.

35. Lundh M, Christensen D, Nielsen MD, et al: Histone deacetylases 1 and 3 but not 2 mediate cytokine-induced beta cell apoptosis in INS-1 cells and dispersed primary islets from rats and are differentially regulated in the islets of type 1 diabetic children. Diabetologia 55: 2421-2431, 2012.

36. Chen LF, Fischle W, Verdin E and Greene WC: Duration of nuclear NF- $\kappa \mathrm{B}$ action regulated by reversible acetylation. Science 293: 1653-1657, 2001.

37. Yu Z, Zhang W and Kone BC: Histone deacetylases augment cytokine induction of the iNOS gene. J Am Soc Nephrol 13: 2009-2017, 2002.

38. Ashburner BP, Westerheide SD and Baldwin AS: The p65 (RelA) subunit of NF- $\kappa B$ interacts with the histone deacetylase (HDAC) corepressors HDAC1 and HDAC2 to negatively regulate gene expression. Mol Cell Biol 21: 7065-7077, 2001.

39. Barter MJ, Pybus L, Litherland GJ, et al: HDAC-mediated control of ERK-and PI3K-dependent TGF- $\beta$-induced extracellular matrix-regulating genes. Matrix Biol 29: 602-612, 2010

40. Lv WW, Wei HM, Wang DL, Ni JQ and Sun FL: Depletion of histone deacetylase 3 antagonizes PI3K-mediated overgrowth of Drosophila organs through the acetylation of histone $\mathrm{H} 4$ at lysine 16. J Cell Sci 125: 5369-5378, 2012.

41. Hargreaves DC, Horng T and Medzhitov R: Control of inducible gene expression by signal-dependent transcriptional elongation. Cell 138: 129-145, 2009.

42. Modak R, Mitra SD, Krishnamoorthy P, et al: Histone H3K14 and H4K8 hyperacetylation is associated with Escherichia coliinduced mastitis in mice. Epigenetics 7: 492-501, 2012.

43. Tsaprouni LG, Ito K, Powell JJ, Adcock IM and Punchard N: Differential patterns of histone acetylation in inflammatory bowel diseases. J Inflamm 8: 1, 2011.

44. Ito K, Barnes PJ and Adcock IM: Glucocorticoid receptor recruitment of histone deacetylase 2 inhibits interleukin-1 $\beta$-induced histone $\mathrm{H} 4$ acetylation on lysines 8 and 12. Mol Cell Biol 20: 6891-6903, 2000.

45. Lowenstein CJ, Alley EW, Raval P, et al: Macrophage nitric oxide synthase gene: two upstream regions mediate induction by interferon gamma and lipopolysaccharide. Proc Natl Acad Sci USA 90: 9730-9734, 1993.

46. Yu Z and Kone BC: Targeted histone $\mathrm{H} 4$ acetylation via phosphoinositide 3-kinase-and p70s6-kinase-dependent pathways inhibits iNOS induction in mesangial cells. Am J Physiol Renal Physiol 290: F496-F502, 2006. 